\title{
Impact of Detector Efficiency and Energy Resolution on Gamma-Ray Background Rejection in Mobile Spectroscopy and Imaging Systems
}

\author{
Timothy J. Aucott ${ }^{\mathrm{a}}$, Mark S. Bandstra ${ }^{\mathrm{b}}$, Victor Negut ${ }^{\mathrm{a}}$, Joseph C. Curtis ${ }^{\mathrm{a}}$, Ross E. Meyer ${ }^{\mathrm{b}}$, Daniel H. Chivers ${ }^{\mathrm{b}}$, Kai Vetter ${ }^{\mathrm{a}, \mathrm{b}}$ \\ ${ }^{a}$ University of California, Berkeley, Department of Nuclear Engineering, Berkeley, CA \\ ${ }^{b}$ Lawrence Berkeley National Laboratory, Nuclear Science Division, Berkeley, CA
}

\begin{abstract}
The presence of gamma-ray background significantly reduces detection sensitivity when searching for radioactive sources in the field, and the systematic variability in the background will limit the size and energy resolution of systems that can be used effectively. An extensive survey of the background was performed using both sodium iodide and high-purity germanium. By using a bivariate negative binomial model for the measured counts, these measurements can be resampled to simulate the performance of a detector array of arbitrary size and resolution. The response of the system as it moved past a stationary source was modeled for spectroscopic and coded aperture imaging algorithms and used for source injection into the background. The performance of both techniques is shown for various sizes and resolutions, as well as the relative performance for sodium iodide and germanium. It was found that at smaller detector sizes or better energy resolution, spectroscopy has higher detection sensitivity than imaging, while imaging is better suited to larger or poorer resolution detectors.
\end{abstract}

Keywords: Background radiation, Gamma-ray detection, Gamma-ray spectroscopy, Gamma-ray imaging

\section{Introduction}

When searching for radioactive sources in the field, detection sensitivity is limited by the presence of the gamma-ray background. Often, a detector system is brought into the field and operated in a background that may not be well understood. This background not only adds statistical noise to detection measurements, but can also vary systematically as the detector moves from one location to the next. Even in environments which have been previously measured, variations in weather or construction of new buildings can change the characteristics of the background. Failure to account for this systematic variability in the background will result in higher false alarm rates than would otherwise be expected.

This variability will limit the size of detectors that can be used effectively in the field, as larger detector arrays suffer more from the systematic uncertainty in the background [1], so sim-

Email address: Timothy . Aucott@SRS.gov (Timothy J. Aucott) ply adding detection efficiency will not necessarily increase detection sensitivity [2]. Similarly, lower resolution detectors will not only suffer from poor statistical certainty [3], but are also less able to resolve source lines from nearby background lines which have large variabilities. In order to successfully detect weak sources in a wide-area search scenario, detectors must incorporate some method to account for the background variability.

This work evaluates the impact of detector efficiency and energy resolution on the ability of systems to detect sources in the field, and it also aims to characterize the difference between the statistical and systematic sources of error. In particular, two approaches are used which have been shown to reduce the systematic uncertainty: spectroscopy and coded aperture imaging [4-6]. The extent of the background variability will determine the relative performance of these two techniques at different resolutions and efficiencies. Spectroscopy will typically have a higher efficiency than an imager, as events in an imager are lost 
to either collimation or event selection. However, spectroscopy systems are also more susceptible to systematic variability, especially in low-resolution detectors.

In both of these cases, the emphasis is on improved detection sensitivity. Both approaches can reduce the uncertainty in the background by looking for a feature in some reduced space. For example, spectroscopy might look for a photopeak in energy space, while imaging looks for a peak pixel in the image space. As these approaches are made more and more sophisticated, they gain the ability to perform more detailed imaging or spectroscopic analysis of the source in question. Imaging, for instance, can provide information on the location or the spatial distribution of the source, while spectroscopy can determine the isotopic composition. However, for purposes of this work, both methodologies are important only in that they also increase detection sensitivity.

The goal of this work is not necessarily to demonstrate the optimum detector or algorithm, but rather to introduce a framework for understanding the background and its effects on various systems. By measuring and characterizing the background over a wide range of locations, the performance of these systems can be measured for a given scenario. The scenario chosen here is the detection of a source of a given isotope, strength, and standoff $\left(1 \mathrm{mCi}\right.$ of ${ }^{137} \mathrm{Cs}$ at $\left.100 \mathrm{~m}\right)$ at a given specificity (one false alarm per hour); this approach, however, can be used to analyze any desired system or scenario.

\section{Experimental Method}

Background measurements were taken with the Radiological Multi-sensor Analysis Platform (RadMAP), a truck-mounted system which consists of 24 high-purity germanium (HPGe) detectors, each $100 \%$ relative efficiency, and a $1 \mathrm{~m}^{2}$ array of sodium iodide $(\mathrm{NaI})$ detectors behind a $2 \mathrm{~cm}$ thick lead coded aperture [5, 7]. The gamma-ray events are acquired in list mode and analyzed in post-processing. Also included are global positioning (GPS) and inertial navigation (INS) systems and the truck speedometer. The RadMAP system was driven around the San Francisco bay area extensively over the past two years; the particular data used for this work were taken between March 13 and May 2, 2012, for a total of 85.5 hours and $2350 \mathrm{~km}$ of background data. The areas surveyed include downtown Oakland and San Francisco, the port of Oakland, suburban and rural areas, and a number of bridges, providing a wide variability of background rates. In most locations, the same route was driven daily in order to provide multiple measurements. A picture of the RadMAP vehicle is shown in figure 1.

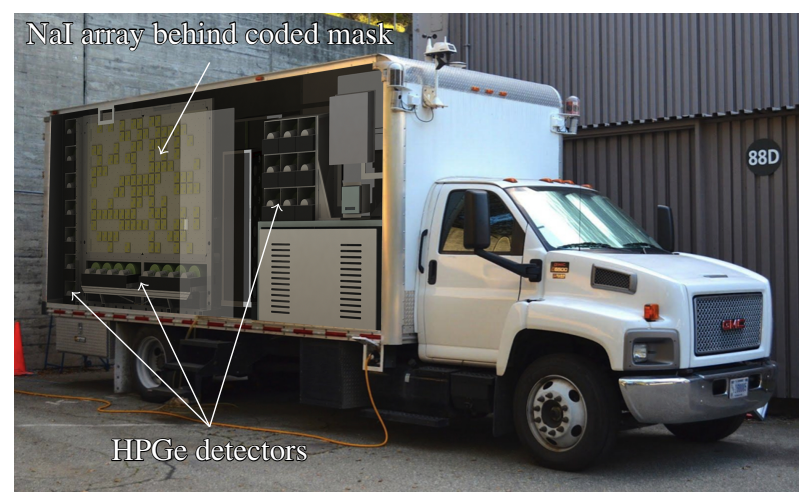

Figure 1: Photograph of the RadMAP vehicle overlaid with a CAD diagram showing the NaI and HPGe detectors inside.

Next, the detector response to a unshielded source was simulated so that source counts could be injected into the measured background runs. The modeling was performed with the SWORD software package [8,9], and responses were simulated for four source nuclides: ${ }^{57} \mathrm{Co},{ }^{131} \mathrm{I},{ }^{137} \mathrm{Cs}$, and ${ }^{60} \mathrm{Co}$. These simulations were performed to account for air attenuation, shielding material in the body of the truck, and the finite size of the detectors and mask elements, and were benchmarked to calibration runs with ${ }^{137} \mathrm{Cs}$. Simulations and benchmarks were performed both with the mask in place (for the imaging analysis) and without the mask (for spectroscopy). For the results presented here, the source was chosen to be $1 \mathrm{mCi}$ of ${ }^{137} \mathrm{Cs}$, placed $100 \mathrm{~m}$ from the road, with the truck moving $25 \mathrm{mph}$. The source was injected over $200 \mathrm{~m}$ (corresponding to the field of view of the coded aperture of $90^{\circ}$ ) with a spatial resolution of $12.5 \mathrm{~m}$ (the imaging resolution of the coded aperture is $25 \mathrm{~m}$ at a $100 \mathrm{~m}$ distance). The algorithms are not required to identify or localize the source; the detection scenario is simply to report the presence or absence of a ${ }^{137} \mathrm{Cs}$ source at a pre-determined location. 
A total of 1655 individual runs were selected from the data set, and the list mode event data were binned into these individual runs to create a distribution of measured backgrounds. Runs were each $200 \mathrm{~m}$ long and required that the vehicle was moving in a straight line and under $25 \mathrm{mph}$, giving at least 18 seconds of background data per run. These parameters were chosen to give a higher likelihood of finding any systematic variability in the imaging background. In most cases, the truck is not traveling exactly $25 \mathrm{mph}$, and so the majority of the runs contain more than 18 seconds of data. These runs must be appropriately downsampled, otherwise the statistical certainty of these measurements will be overestimated. To accomplish this, each event in the run has some probability $p$ of being selected to remain in the run. For example, if the truck were traveling only $20 \mathrm{mph}$, then each gamma-ray event in the run would have a one-in-five chance of being removed from the data set.

It is noted that this random selection criterion is the same as a binomial sampling process. For a run initially containing $n$ events, the probability mass function for the remaining events $k$ in the downsampled run is simply the binomial distribution:

$$
P_{\mathrm{B}}(k ; n, p)=\left(\begin{array}{l}
n \\
k
\end{array}\right) p^{k}(1-p)^{n-k}
$$

where the probability $p$ is the desired time (here 18 seconds) over the actual run time. This binomial downsampling method can then be used without having list mode data, and it can also be used to model the count rate in a lower-efficiency detector. For example, in order to reduce the efficiency of a detector array by $50 \%$, the probability $p$ can be set to 0.5 and number of counts $n$ can be resampled according to equation 1 .

\section{Spectroscopy and Imaging Approaches}

Spectroscopy and imaging were used in order to reduce the systematic uncertainty in the background. The two algorithms operate by making a measurement of the source signal in some reduced space, whether in the spectrum or the reconstructed image. Both algorithms also take a measurement of the background in order to estimate the background in the region of interest. For the case of spectroscopy, neighboring regions of the spectrum are used for the background estimate, while for the coded aperture, the background estimate is made by the detectors which are shielded from the source by the mask. Spectroscopy was performed with both the NaI and HPGe detectors, while the imaging was performed only with the $\mathrm{NaI}$, as these were the only detectors behind the coded mask.

The spectroscopy algorithm integrates the counts in a window $\pm \sqrt{2} \times \sigma_{E}$ wide, where $\sigma_{E}$ is the peak width at the energy of interest, and estimates the background using two side windows, each half as wide. For example, when looking for ${ }^{137} \mathrm{Cs}$ with the NaI array, the primary window is at $629-695 \mathrm{keV}$, while the side windows are at 533-566 and 758-791 keV [10]. The coded aperture uses the same peak window, but uses a balanced decoding matrix to create a background-subtracted image [11]. The source spectra from the simulations were Poisson sampled to correspond to a $1 \mathrm{mCi}{ }^{137} \mathrm{Cs}$ source at a distance of $100 \mathrm{~m}$ from the vehicle, and were then injected into the background data.

After measuring the background distribution and injecting the appropriate source counts, receiver operator characteristic (ROC) curves were generated for both the spectroscopy and imaging approaches. These plots allow different detection algorithms to be compared across all true and false positive rates [12]. Example ROC curves are shown in figure 2 for the spectroscopy and imaging algorithms in the $\mathrm{NaI}$ array and three of the HPGe detectors.

In order to compare the ROC curves at different resolutions and efficiencies, a constant false alarm of once per hour was set as the reference threshold, and the detection rates at this threshold will be reported for both algorithms. If only one energy and image location is being searched at a time, this corresponds to a $0.5 \%$ false alarm probability. This assumption is made in order to make a fair comparison between algorithms. A real-world system would of course have to alarm on many isotopes and locations, but then the performance depends on the exact list of isotopes and range of distances that need to be detected. Note that the large number of background measurements allow for the performance to be evaluated at even lower false alarm rates. 


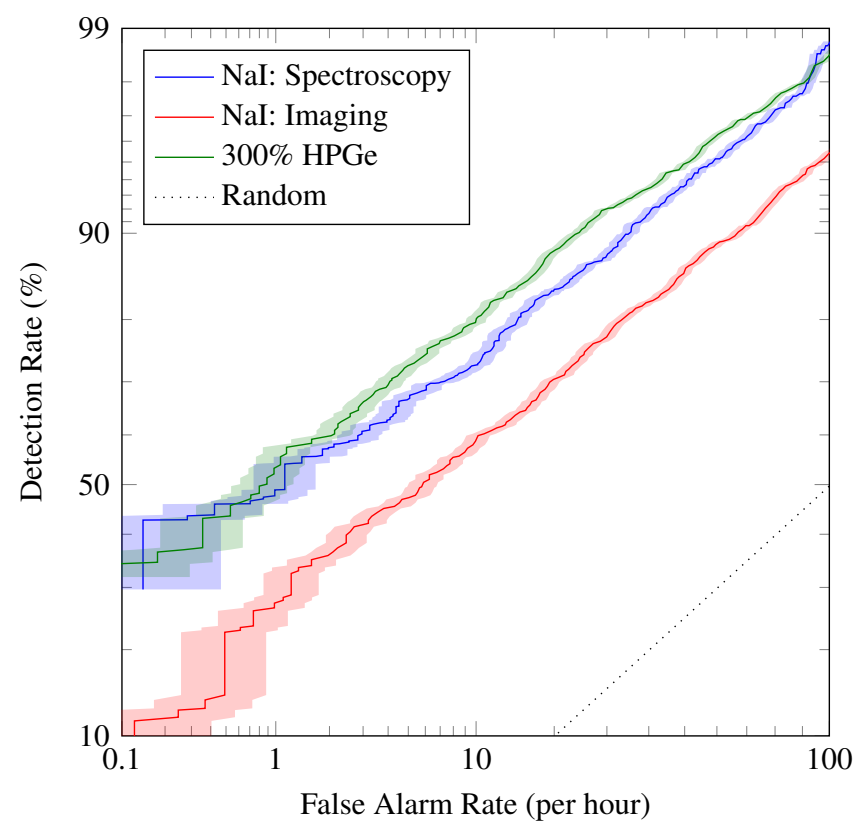

Figure 2: ROC curves for detecting $1 \mathrm{mCi}$ of ${ }^{137} \mathrm{Cs}$ at $100 \mathrm{~m}$ standoff using the $1 \mathrm{~m}^{2} \mathrm{NaI}$ array for both spectroscopy and imaging algorithms, as well 3 HPGe (each $100 \%$ relative efficiency) for comparison. Both axes are plotted on a normal deviate scale. Shaded regions indicate $68 \%$ confidence intervals.

Due to the high resolution of the HPGe, there are very few background counts in the source window, allowing for good detection without interference from the background. For example, when comparing spectroscopy results between the two detector types, $300 \%$ relative efficiency of germanium gives the same detection performance as the full $1 \mathrm{~m}^{2}$ sodium iodide array using the linear algorithm described here, although this comparison is highly dependent on the particular algorithms used for the $\mathrm{NaI}$ data. By using background training sets [10], the sodium iodide could achieve the same performance as at least $700 \%$ relative efficiency of germanium.

\section{Statistical Model}

In order to understand the extent of the systematic variability, an appropriate model must be used to characterize the distribution of the background flux. By histogramming the count rates $y$ in the photopeak window (that is, with no imaging or spectroscopy applied), a measured probability distribution $P_{\mathrm{m}}(y)$ can be created as shown in figure 3 . This distribution illustrates two important features of the background. First, the variance is much wider than would be expected from assuming a Poisson distribution, which has a single parameter $\lambda$, corresponding to both the mean and the variance:

$$
P_{\mathrm{P}}(y ; \lambda)=\frac{\lambda^{y}}{y !} \frac{1}{e^{\lambda}} .
$$

Instead, the observed distribution is seen to be overdispersed by a factor of over 100 in variance, which reflects the systematic uncertainty introduced by the background. Second, the distribution is tailed towards higher rates, whereas the Poisson distribution is nearly symmetric at these count rates.

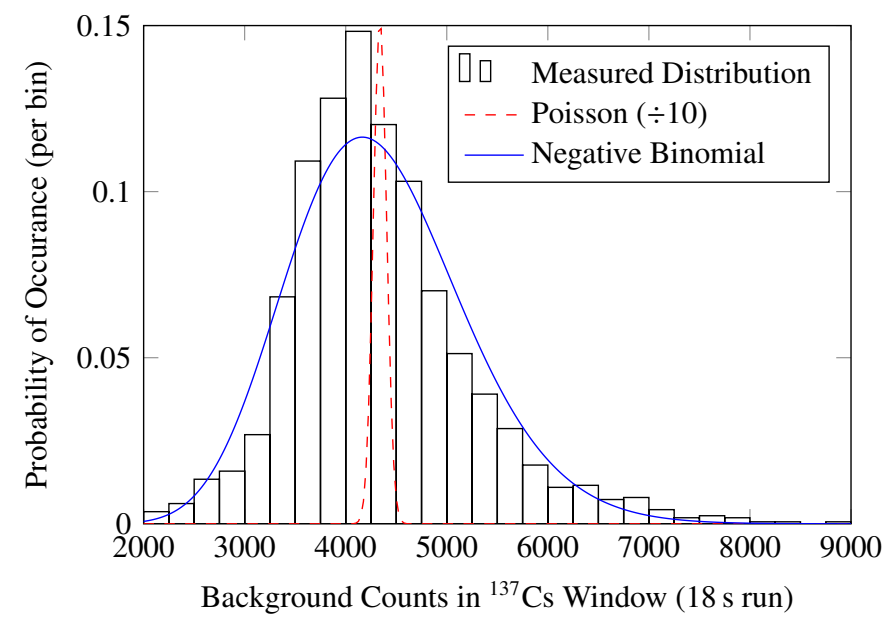

Figure 3: Background distribution in the ${ }^{137} \mathrm{Cs}$ photopeak window for $200 \mathrm{~m}$, $18 \mathrm{~s}$ runs. The dashed distribution corresponds to the Poisson distribution, while solid distribution is the negative binomial. The Poisson distribution is plotted at a reduced vertical scale for visibility.

Instead of a Poisson distribution, the shape of the background distribution can be modeled as a negative binomial distribution [13] in order to account for the overdispersion:

$$
P_{\mathrm{NB}}(y ; r, \lambda)=\frac{\lambda^{y}}{y !} \frac{1}{\left(1+\frac{\lambda}{r}\right)^{r}} \frac{\Gamma(r+y)}{\Gamma(r)(r+\lambda)^{y}} .
$$

This formulation introduces an additional parameter $r$ that increases the variance without changing the mean of the distribution. The mean remains $\lambda$ while the variance can be expressed as

$$
\sigma_{\mathrm{NB}}^{2}=\lambda+\frac{\lambda^{2}}{r}
$$

which allows the variance to be decomposed into two components. The first, $\lambda$, is usual Poisson variance, while the second, 
$\lambda^{2} / r$, is the additional systematic variance, and when $r \rightarrow \infty$, the negative binomial reduces to the Poisson case. This model will be further refined for the case of the imaging and spectroscopy count rates.

Figure 3 reveals that the two-parameter model does not perfectly fit every feature of the background distribution. The primary reasons are likely that the measurements cannot sample all possible environments, as well as the fact that the environments were not sampled in a perfectly uniform manner. For instance, downtown areas were measured daily due to their proximity, while rural areas were measured less frequently. Others who have measured the background distribution [4] also identified strong systematic variability, but the exact distribution will depend highly on the variety of environments sampled.

\section{Detector Efficiency}

Ideally, one would like to know the performance of an arbitrarily large detector in the field, in order to evaluate if the increase in detection is worth the cost. However, any measurements have systematic variations in the underlying flux, blurred by the Poisson statistics inherent in a real detector. This means that simply scaling the measured count rate will not accurately reflect the true contributions from the systematic and statistical spread. Furthermore, spectroscopy and imaging algorithms make an additional measurement of the background that is highly correlated to the source measurement. The challenge is to understand the underlying flux distribution that is emitted by the environment, before any Poisson noise is introduced.

One solution is to model the measured count rates $y$ as Poisson distributed, but that the flux $x$ varies according to the gamma distribution [14]:

$$
P_{\mathrm{G}}(x ; r, \lambda)=\frac{\left(\frac{r}{\lambda}\right)^{r}}{\Gamma(r)} x^{r-1} e^{\frac{-x r}{\lambda}} .
$$

This formulation is chosen for the underlying flux because the resulting Poisson mixture $P(y)$ then follows the negative binomial distribution as in equation 3 .

This matches the observed distribution for the background counts in a single channel, but for spectroscopy and imaging, the algorithms output the difference between two correlated, Poisson distributed channels. In order to model this, a bivariate negative binomial model is used [15]. Each channel (one for source-plus-background, the other for the background estimate) is modeled as the sum of two gamma distributions:

$$
X_{i}=U+V_{i} ; \quad i=1,2
$$

where the distribution $U$ is common to both, representing the positively correlated component, and $V_{i}$ represents the uncorrelated component. The correlated term $U$ is dominant and corresponds to $P_{G}(r, \lambda)$ in equation 5 . The uncorrelated terms are also chosen to be gamma functions $P_{G}\left(r_{i}, \lambda_{i}\right)$, except with their parameters $r_{i}$ and $\lambda_{i}$ unknown. Each channel is then Poisson sampled, and then the background estimate is subtracted from the source-plus-background measurement to create the final output. The four unknown parameters $\left(r_{1}, r_{2}, \lambda_{1}, \lambda_{2}\right)$ are found via a least-squares fit to the measured data.

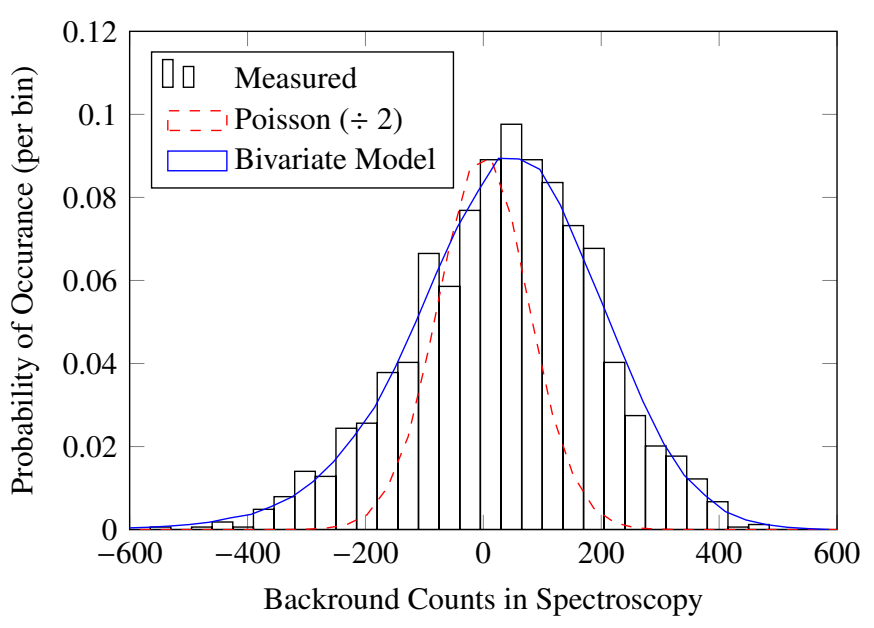

Figure 4: Background distribution in the ${ }^{137} \mathrm{Cs}$ spectroscopy algorithm for $200 \mathrm{~m}, 18 \mathrm{~s}$ runs. The dashed distribution corresponds to the case with Poisson distribution, while the solid distribution is the bivariate model. The Poisson distribution is plotted at a reduced vertical scale for visibility.

Figure 4 shows the measured distribution of background counts in the spectroscopy algorithm. If the spectral regions were perfectly correlated, the spectroscopy output would only exhibit Poisson error (dashed line); this corresponds to the case where $V_{i}=0$. The fit to the distribution (solid line), however, indicates that there is still some systematic uncertainty in the 
algorithm, which contributes to the broader width and tailing at the lower end. The width is still much smaller than the gross photopeak window counts (shown in figure 3), but potentially could be improved by using more advanced approaches.

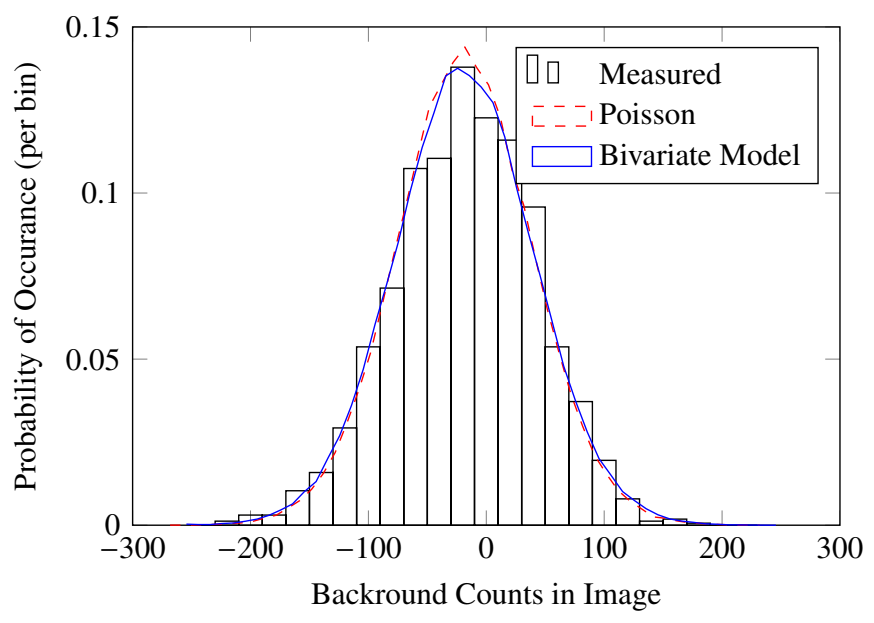

Figure 5: Background distribution in the ${ }^{137} \mathrm{Cs}$ imaging algorithm for $200 \mathrm{~m}$, $18 \mathrm{~s}$ runs. The dashed distribution corresponds to the case with Poisson noise only, while the solid distribution is the bivariate model.

On the other hand, figure 5 shows the distribution of counts in the imaging algorithm. In this case, the Poisson assumption is quite close to the measured distribution, and the bivariate model is not significantly better than the Poisson case. This indicates that the two channels (masked and unmasked) in the coded aperture are well correlated, and that, at least in this data set, there were no observed systematic features in the image algorithm. This is consistent with the imaging distributions measured by others, which closely follow a Gaussian distribution [4], again indicating no systematic variability. The disadvantage of the imaging algorithm, however, is that the required collimation reduces the source intensity by at least $50 \%$.

Once these parameters are found, this model allows for the flux distributions to be scaled to any desired detector efficiency before the Poisson noise is applied. This allows the performance of the detection algorithms to be extrapolated to larger detector sizes while preserving the correct Poisson statistics, as long as the systematic variance has been described adequately by the fit. The detection sensitivity can then be evaluated for the desired false alarm rate, in this example one false alarm per hour. The result of this scaling is shown in figure 6, which applies the spectroscopy and imaging algorithms to a $\mathrm{NaI}$ array of varying size. The data points indicate the measured detection rates for the actual system $\left(1 \mathrm{~m}^{2}\right)$, as well as for some subsamples of the array. This was accomplished by downsampling the counts in each detector by randomly sampling according to equation 1.

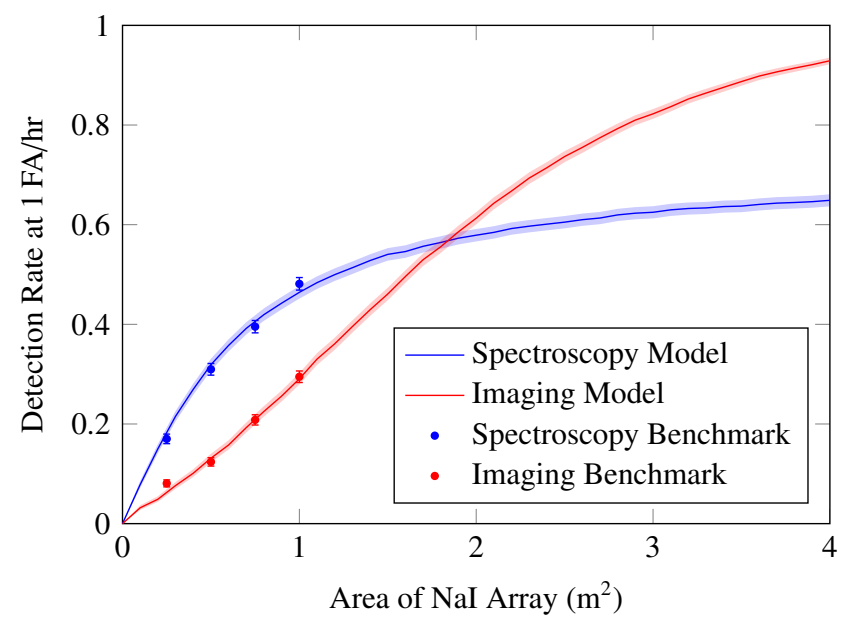

Figure 6: Detection rates for detecting $1 \mathrm{mCi}$ of ${ }^{137} \mathrm{Cs}$ at a standoff of $100 \mathrm{~m}$ with a NaI array of varying size. Data points are the actual system measurements, while the lines indicate the bivariate negative binomial model. Shaded regions indicate $68 \%$ confidence intervals.

The spectroscopy algorithm is unable to completely account for the background variability, and so the detection rate saturates below $70 \%$. This is represented in the bivariate model by the lack of perfect correlation between the peak region and the background regions (the correlation for this spectroscopy algorithm was measured to be 0.98 ). More advanced algorithms, for example, ones which train on previously measured data, may be able improve the maximum performance, but unless an algorithm can achieve perfect correlation, the maximum detection rate will always be limited to some value less than 1 .

The coded aperture algorithm suffers from fewer statistics due to the presence of the mask (as well as other effects such as attenuation through the mask and blurring due to the motion of the truck), but was able to completely compensate for the background variability. In this background data set, the counts in the masked and unmasked detectors were perfectly correlated 
to within the statistical certainty of the measurements. This means that although the imaging performance was poorer than spectroscopy for this system, above $1.8 \mathrm{~m}^{2}$ the coded aperture performs better than spectroscopy.

\section{Energy Resolution}

In order to model the effects of energy resolution, the measured HPGe source and background spectra were convolved with a Gaussian kernel with a given width. The HPGe had an energy resolution of $0.3 \%$ FWHM at $662 \mathrm{keV}$, so the kernel width was chosen such that when the kernel width is added in quadrature with the HPGe width, the desired resolution is achieved:

$$
\sigma_{\text {kernel }}^{2}=\sigma_{\text {desired }}^{2}-\sigma_{H P G e}^{2} .
$$

The resulting source spectra are shown in figure 7 for a range of energy resolutions.

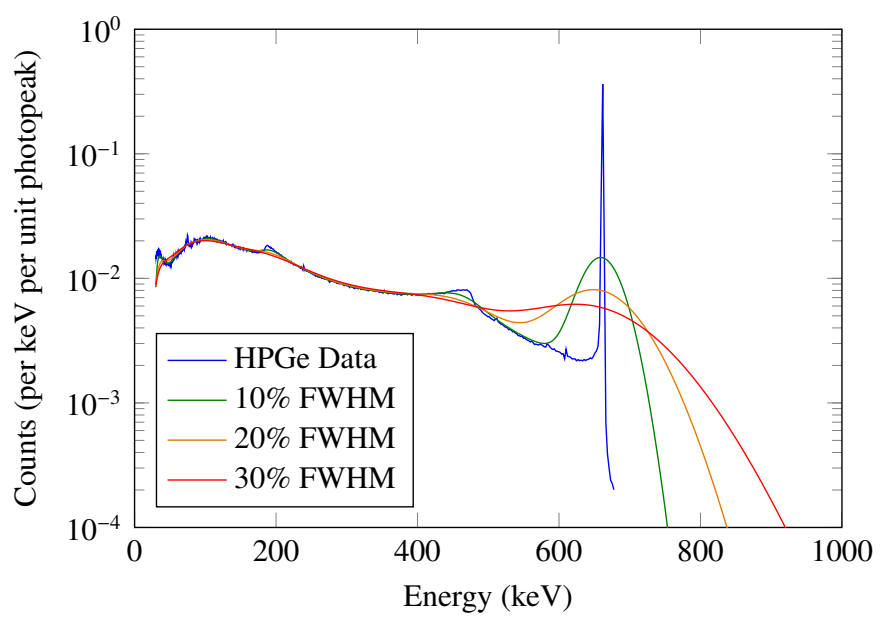

Figure 7: Measured ${ }^{137}$ Cs spectrum in HPGe and blurred spectra with 10, 20, and 30\% FWHM at the photopeak energy (662 keV). Spectra are normalized so that the integral of the photopeak is one.

In order to compare the HPGe array to the NaI array (which has a much larger area), the HPGe data was scaled up in efficiency as demonstrated in the section 5 in order to match the $\mathrm{NaI}$ array size. It is assumed that the variance in the HPGe array can be well measured because of its high energy resolution. The relative efficiencies of the two arrays at $662 \mathrm{keV}$ was obtained during the benchmarking measurements with ${ }^{137} \mathrm{Cs}$, al- though application to other energies will require separate measurements of efficiency, due to the difference in intrinsic efficiencies between NaI and HPGe. The impact of energy resolution on the two detection approaches is shown in figure 8 .

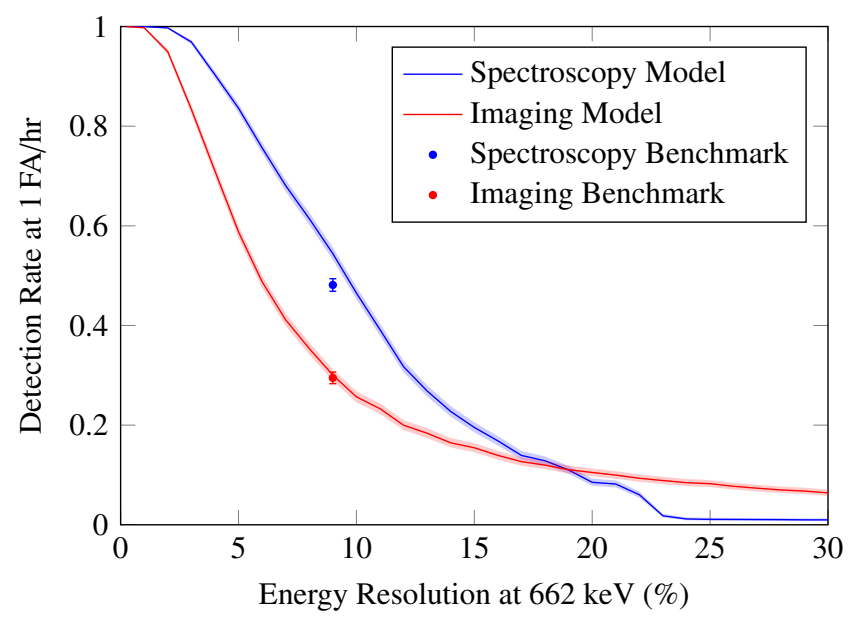

Figure 8: Detection rates for detecting $1 \mathrm{mCi}$ of ${ }^{137} \mathrm{Cs}$ with a $1 \mathrm{~m}^{2}$ array of varying energy resolutions. Data points are the actual system measurements made with NaI, while the lines indicate model results. Shaded regions indicate $68 \%$ confidence intervals.

As the resolution becomes broader, spectroscopy shows an increase in both statistical and systematic noise, resulting in an increased drop above 15\% FWHM as background lines (e.g., the $609 \mathrm{keV}$ line from the radon daughter ${ }^{214} \mathrm{Bi}$ ) are unable to be resolved from the source. At worse resolutions, the peak can no longer be seen by the spectroscopy algorithm; this is due to the fact that the peak is not resolvable from the Compton edge, as seen in figure 7.

Because the imaging algorithm sets an energy window, increasing the peak width also increases the width of this window and adds some statistical noise, hence the decreased detection rate at reduced resolutions. This is important because an imaging system that images a wider region of the spectrum will accumulate unnecessary statistical noise. At better energy resolutions, the coded aperture does not perform as well as spectroscopy due to fewer statistics introduced by the mask, but it is still able to create an image and detect the source at very poor resolutions, giving better performance than spectroscopy above $18 \%$ FWHM at $662 \mathrm{keV}$. 
From figure 8, it can be seen that the energy resolution of the detectors in this scenario is at least as important as the difference between spectroscopy and imaging, and either approach would benefit from having better spectral knowledge. For instance, imaging has the advantage of being able to detect localized sources in the presence of an ambient background of the same isotope. In this case, improved resolution would still reduce the statistical noise from the rest of the background and improve detection. Another advantage of imaging is its flexibility in setting wide energy windows, for instance, when searching for shielded sources where the photopeak is downscattered to lower energies. In this case, the advantage of resolution would likely be lost.

Although the HPGe is not an imaging system (the coded aperture is only in front of the NaI array), the findings in section 5 indicate that there are not significant systematic variances in the imaging algorithm. This allows a coded aperture system to be approximated by randomly assigning $50 \%$ of the germanium counts to the unshielded pixels and the remainder to the shielded pixels, and then using the same reconstruction algorithm as for the sodium iodide.

\section{Conclusions}

In many search scenarios, detection systems will be limited by the systematic variations in the background, not necessarily the statistical uncertainty, and it is important to understand the effects of both. This is relevant not only when evaluating the performance of these systems, but also when designing a new system. In particular, systems which use large volumes of lowresolution detectors will be affected by the relative contribution of both the systematic and statistical uncertainty. This problem is particularly important in applications which require low false alarm rates, as systematic uncertainties will create much broader and more tailed distributions than anticipated, resulting in more false alarms than otherwise expected.

A large set of background measurements is used for source injection in order to evaluate the performance for a given scenario. By making extensive measurements of the gamma-ray background with both HPGe and NaI, their performances can be compared by measuring the detection rate for a subset of HPGe detectors. For example, when using spectroscopy, 300\% relative efficiency of germanium gives the same detection performance as the full $1 \mathrm{~m}^{2}$ sodium iodide array using the linear algorithm described here. By using background training sets [10], the sodium iodide can achieve the same performance as $700 \%$ relative efficiency of germanium. Because of their high resolution, there are very few background counts in the source window, allowing for good detection as long as the background does not contain the exact isotope in question. For these data, there were no interferences for the isotopes studied (the ${ }^{137} \mathrm{Cs}$ fallout is much too weak in California to see in 18 seconds). Although not present on this platform, an HPGe imager could offer the benefit of high resolution when imaging is required (for example, if the isotope in question is present in the background).

Having these measurements allows the background rates to be characterized more thoroughly beyond the simple Poisson assumption. By using a model which treats the underlying flux as gamma distributed, the systematic and statistical sources of noise can be quantified separately. Next, the response of more sophisticated algorithms such as spectroscopy and imaging algorithms can be modeled using a bivariate negative binomial distribution. These models fit well to the measured background distributions created with the two algorithms, and there are analytic functions available to describe both the measured rates and the underlying flux with only a few required parameters. By using this model, the detection sensitivity can be evaluated for a system of arbitrary size and resolution. Spectroscopy performed better than the coded aperture for the scintillator system on board $\left(1 \mathrm{~m}^{2}\right.$ of $\left.\mathrm{NaI}\right)$, but with a larger array $\left(>1.8 \mathrm{~m}^{2}\right)$ or poorer resolution detectors ( $>18 \%$ FWHM at $662 \mathrm{keV})$, imaging will be better able to compensate for background variability.

Finally, this approach provides useful tools for further understanding of the background and development of detection systems. The primary focus of these results is not necessarily to demonstrate the best possible performance, but to show how 
different concepts can be evaluated and understood in a complex background environment. The data collected and exhibited here provide the ability to study detection concepts at the relevant false alarm rates of once per hour or less. This framework can also be used to study other detection scenarios beyond the example shown here, including detection of other isotopes or shielded source scenarios.

\section{Acknowledgment}

The authors would like to thank Ren Cooper, Lucian Mihailescu, Donald Gunter, Michael Quinlan, Brian Quiter, and Brian Plimley for their help in preparing this material. We are grateful to Luster Howard, Shelley Caras, Robert Kelley, Paul Williams, and Jon Cleveland from the Lawrence Berkeley Lab facilities team for driving the RadMAP vehicle. The engineering team at the Lawrence Berkeley Lab were also extremely supportive in their maintenance of the vehicle. The authors also thank the team from the Naval Research Lab for designing, building, and providing training for the original MISTI system.

This work was supported by the U.S. Department of Homeland Security under Grant Award 2011-DN-077-ARI049-03. The modeling and data curation were performed with the assistance of the National Energy Research Scientific Computing Center, which is supported by the Office of Science of the U.S. Department of Energy under Contract No. DE-AC0205CH11231. This support does not constitute an express or implied endorsement on the part of the government.

\section{References}

[1] K. P. Ziock, K. E. Nelson, Maximum detector sizes required for orphan source detection, Nuclear Instruments and Methods in Physics Research Section A: Accelerators, Spectrometers, Detectors and Associated Equipment 579 (1) (2007) 357-362.

[2] K. P. Ziock, The lost source, varying backgrounds and why bigger may not be better, in: J. I. Trombka (Ed.), Unattended Radiation Sensor Systems for Remote Applications,, Vol. CP632, American Institute of Physics, 2002, pp. 60-70.

[3] G. Armantrout, A. Bradley, P. Phelps, Sensitivity problems in biological and environmental counting, Nuclear Science, IEEE Transactions on 19 (1) (1972) 107-116. doi:10.1109/TNS.1972.4326494.
[4] K. P. Ziock, J. W. Collins, L. Fabris, Source-search sensitivity of a largearea, coded-aperture, gamma-ray imager, IEEE Transactions on Nuclear Science 53 (3) (2006) 1614-1621.

[5] L. Mitchell, B. Phlips, Mobile Imaging and Spectroscopic Threat Identification (MISTI): System overview, in: Nuclear Science Symposium Conference Record (NSS/MIC), 2009 IEEE, 2009, pp. 110-118. doi:10.1109/NSSMIC.2009.5401849.

[6] M. V. Hynes, M. Toolin, B. Harris, The Raytheon-SORDS trimodal imager, in: Proc. SPIE, Vol. 7310, 2009, pp. 731003-731003-7. doi: $10.1117 / 12.818735$.

[7] T. J. Aucott, Gamma-ray background variability in mobile detectors, Ph.D. thesis, University of California, Berkeley (Fall 2014).

[8] E. Novikova, M. Strickman, Designing SWORD-SoftWare for Optimization of Radiation Detectors, in: Nuclear Science Symposium Conference Record, 2006. IEEE, Vol. 1, 2006, pp. 607-612. doi:10.1109/NSSMIC.2006.356228.

[9] J. C. Curtis, Benchmarking the gamma-ray response of the radiological multi-sensor benchmarking the gamma-ray response of the radiological multi-sensor analysis platform, Master's thesis, University of California, Berkeley (Fall 2014).

[10] T. Aucott, M. Bandstra, V. Negut, J. Curtis, D. Chivers, K. Vetter, Effects of background on gamma-ray detection for mobile spectroscopy and imaging systems, Nuclear Science, IEEE Transactions on 61 (2) (2014) 985-991. doi:10.1109/TNS.2014.2306998.

[11] E. E. Fenimore, T. M. Cannon, Coded aperture imaging with uniformly redundant arrays, Applied optics 17 (3) (1978) 337-347.

[12] W. W. Peterson, T. G. Birdsall, W. C. Fox, The theory of signal detectability, in: Proceedings of the IRE Professional Group on Information Theory, Vol. 4, 1954, pp. 171-212.

[13] N. L. Johnson, A. W. Kemp, S. Kotz, Univariate Discrete Distributions, John Wiley \& Sons, 2005.

[14] G. J. Hahn, S. S. Shapiro, Statistical Models in Engineering, John Wiley \& Sons, Inc., 1994.

[15] M. J. Maher, A bivariate negative binomial model to explain traffic accident migration, Accident Analysis \& Prevention 22 (5) (1990) 487-498. 